\title{
Pathogenic STX3 variants affecting the retinal and intestinal transcripts cause an early-onset severe retinal dystrophy in microvillus inclusion disease subjects
}

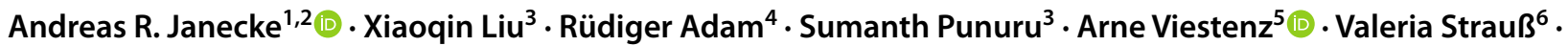 \\ Martin Laass $^{7}$. Elizabeth Sanchez ${ }^{8} \cdot$ Roberto Adachi $^{8}\left(\mathbb{C}^{\circ} \cdot\right.$ Martha P. Schatz $^{9} \cdot$ Ujwala S. Saboo $^{9}$ (1) Naveen Mittal $^{10}$. \\ Klaus Rohrschneider ${ }^{11}$ (D) Johanna Escher ${ }^{12}$ D . Anuradha Ganesh ${ }^{13}$. Sana Al Zuhaibi ${ }^{13} \cdot$ Fathiya Al Murshedi $^{14}$ (D) \\ Badr AlSaleem ${ }^{15}$ (D) Majid Alfadhel ${ }^{16}$ (D) Siham Al Sinani ${ }^{17^{(D)}} \cdot$ Fowzan S. Alkuraya $^{18^{(D)}} \cdot$ Lukas A. Huber $^{19}$ (D) . \\ Thomas Müller $^{1} \cdot$ Ruth Heidelberger $^{3}$ (D) $\cdot$ Roger Janz $^{3,20}$ (iD
}

Received: 17 February 2021 / Accepted: 15 April 2021 / Published online: 11 May 2021

(c) The Author(s) 2021

\begin{abstract}
Biallelic STX3 variants were previously reported in five individuals with the severe congenital enteropathy, microvillus inclusion disease (MVID). Here, we provide a significant extension of the phenotypic spectrum caused by STX3 variants. We report ten individuals of diverse geographic origin with biallelic STX3 loss-of-function variants, identified through exome sequencing, single-nucleotide polymorphism array-based homozygosity mapping, and international collaboration. The evaluated individuals all presented with MVID. Eight individuals also displayed early-onset severe retinal dystrophy, i.e., syndromic —intestinal and retinal—disease. These individuals harbored STX3 variants that affected both the retinal and intestinal STX3 transcripts, whereas STX3 variants affected only the intestinal transcript in individuals with solitary MVID. That STX3 is essential for retinal photoreceptor survival was confirmed by the creation of a rod photoreceptor-specific STX3 knockout mouse model which revealed a time-dependent reduction in the number of rod photoreceptors, thinning of the outer nuclear layer, and the eventual loss of both rod and cone photoreceptors. Together, our results provide a link between STX3 loss-of-function variants and a human retinal dystrophy. Depending on the genomic site of a human loss-of-function STX3 variant, it can cause MVID, the novel intestinal-retinal syndrome reported here or, hypothetically, an isolated retinal dystrophy.
\end{abstract}

\section{Introduction}

A severe form of hereditary diarrhea called microvillus inclusion disease (MVID, OMIM 251850) (Vogel et al. 2016) was described in five individuals with biallelic mutations of the syntaxin 3 gene (STX3, OMIM 600876) by us and by others (Alsaleem et al. 2017; Julia et al. 2019; Wiegerinck et al. 2014). MVID is characterized by a loss of microvilli, microvillus inclusions, and the accumulation of

Ruth Heidelberger and Roger Janz have contributed equally to this work.

Andreas R. Janecke

andreas.janecke@i-med.ac.at

Ruth Heidelberger

ruth.heidelberger@uth.tmc.edu

Extended author information available on the last page of the article subapical vesicles in intestinal epithelial cells, suggestive of a trafficking defect (Vogel et al. 2015, 2017). Syntaxin genes code for N-ethylmaleimide-sensitive factor attachment protein receptor (SNARE) proteins that catalyze the fusion between vesicles and their target membranes (Rizo and Sudhof 2012). A major STX3 spliceform, STX3A, is expressed in enterocytes. STX3 is required for the proper trafficking of vesicles to and fusion with the apical membrane in mammalian epithelial cells (Low et al. 1998; Vogel et al. 2015) indicating that the lack of STX3A in epithelial cells causes MVID. We have characterized another transcript, syntaxin $3 \mathrm{~B}$, generated by differential splicing and highly expressed in the retinas of mice and fish, where syntaxin 3A mRNA is only expressed at very low levels (Curtis et al. 2008, 2010). Stx $3 a$ and Stx3b proteins differ in the C-terminally-located SNARE and transmembrane domains (Fig. 1a). Stx3 localizes to the synaptic boutons of photoreceptors and bipolar cells and to the inner segments of photoreceptors of the 
A

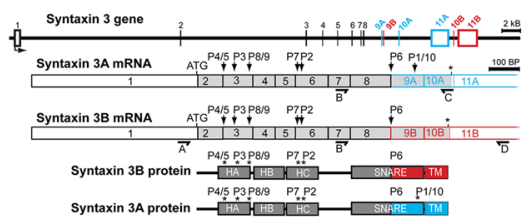

B

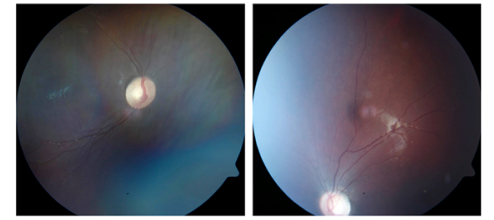

C

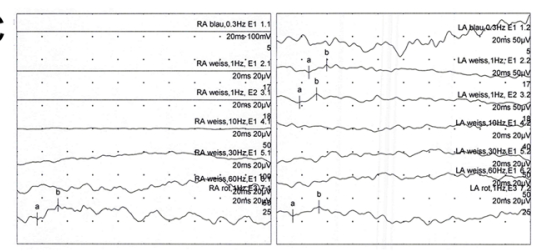

D

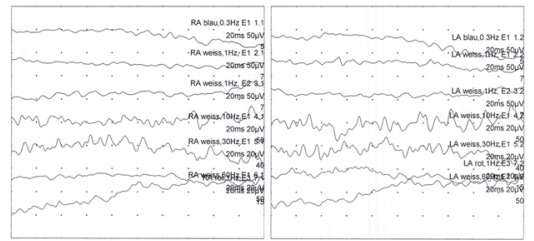

$E$
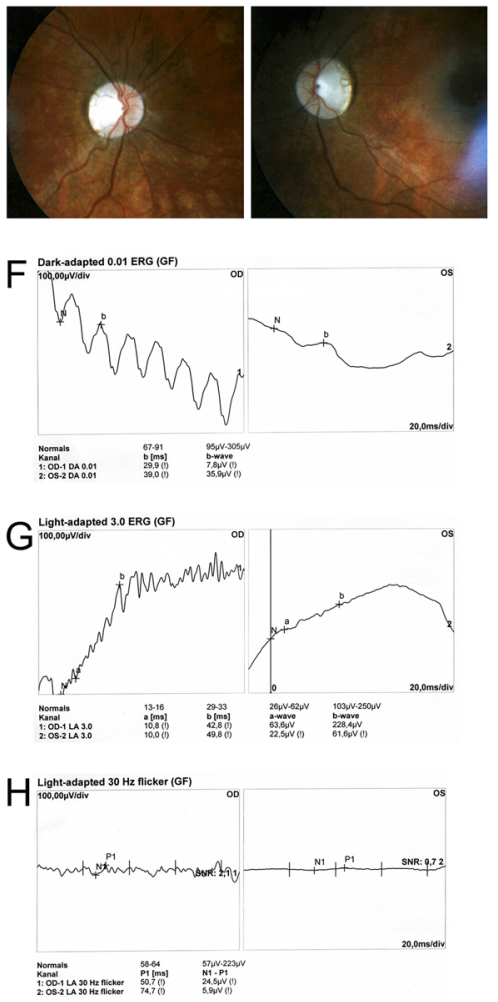

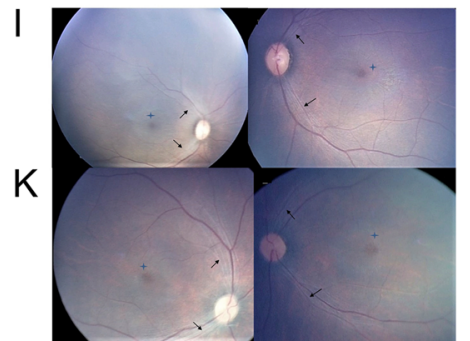

L

M

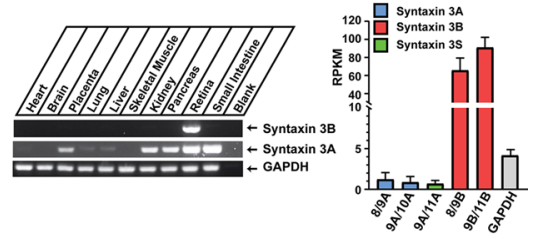

Fig. 1 STX3 structure and human pathogenic variants, the human retinal phenotype, and STX3 tissue expression. a Two major isoforms (STX3A and STX3B) differing in the C-terminal domains are expressed by the STX3 gene; the location of the pathogenic variants in human subjects $\mathrm{P} 1-\mathrm{P} 10$ are indicated. All the pathogenic variants are expected to lead to truncated proteins or nonsense-mediated mRNA decay due to premature stop codons. The pathogenic variant in subjects P1 and P10 only affects the STX3A transcript. Primers used for amplification of the human $S T X 3 B$ mRNA are indicated. b Fundus images of subject P2 at age 10 years. Note the pallor of the optic disk, and mild retinal arteriolar narrowing. Severely reduced amplitudes seen with ERG recordings obtained with skin electrodes in subject P2 at 1 year (c) and at 10 years of age (d); note, the first 3 ERG traces in (c) are flat due to non-recording electrodes. e Fun-

rodent retina (Robichaux et al. 2019). It has been implicated in the trafficking of rhodopsin to the outer segments of rod photoreceptors (Chuang et al. 2007; Mazelova et al. 2009), and in the trafficking of peripherin-2 (RDS, PRPH2, OMIM 179605) and rod outer segment protein 1 (ROM1, OMIM 180721) (Zulliger et al. 2015). Finally, a role of STX3 in the release of neurotransmitter at photoreceptor and bipolar cell synaptic terminals was revealed in non-mammalian vertebrate retina (Curtis et al. 2010; Datta et al. 2017; Hays et al. 2020).

We assembled a cohort of ten individuals in eight families, all with MVID, of whom eight displayed a novel syndrome, consisting of MVID and early-onset severe retinal dystrophy (EOSRD). All affected individuals had dus images of subject P3 at age 5 years. Note the pallor of the optic disk. f-h Selected ERG traces show a completely flat waveform in subject P3 at 5 years of age. Pallor of the optic disks and attenuated nerve fibers (arrows) in subjects P4 (i) and P5 (k). 1 STX3B is a retinal-specific transcript in humans. Primers specific for the STX3A and 3B mRNA were used to analyze the expression in different human tissues. GAPDH was amplified as a control. $\mathbf{m}$ STX3B is the main STX3 isoform expressed in the retina. RNA-sequencing data sets from normal human retina samples were analyzed for the presence of matching reads corresponding to STX3A (exons 8/9A, 9A/10A) and STX3B (exons 8/9B, 9B/10B). Reads per kilobase of transcript, per million mapped reads (RPKM) are shown. (8/9A: $1.2 \pm 0.87,9 \mathrm{~A} / 10 \mathrm{~A}: 0.83 \pm 0.75,9 \mathrm{~A} / 11 \mathrm{~A}: 0.65 \pm 0.44,8 / 9 \mathrm{~B}: 65 \pm 14$, 9B/10B:91 \pm 12 , GAPDH: $4.1 \pm 0.74 ; \mathrm{RPKM} \pm \mathrm{SD},(n=6))$

homozygous loss-of-function STX3 variants, with a correlation between $S T X 3$ genotypes and intestinal and retinal organ involvement. We demonstrate that $S T X 3 B$ is highly expressed in human retina and that the protein is enriched in the inner and outer segments of photoreceptors and in ribbon synapses of the human retina. Finally, we show that the inactivation of Stx3 in murine rod photoreceptors leads to a progressive degeneration of photoreceptors, corroborating a recently published study that used a different Stx3 knockout mouse line (Kakakhel et al. 2020). Our study demonstrates that STX3 is essential for the function of the mammalian retina and that human variants affecting $S T X 3 B$ are associated with retinal dysfunction. 


\section{Materials and methods}

For all clinical data presented including the molecular genetic studies appropriate informed consent was obtained from subjects or their parents in accordance with the guidelines of the respective institutes at which the subjects were seen and approved by the institutional review boards and ethics committees as required. Human cadaver tissue was donated to the Willed Body Program at the McGovern Medical School at the University of Texas Health Science Center in Houston. Use of cadaver specimens is deemed exempt by the Institutional Review Board.

\section{Animals}

Animal procedures conformed to National Institutes of Health guidelines and were approved by the Animal Welfare Committee of the University of Texas Health Science Center at Houston. Mice were kept under standard housing conditions with unlimited access to food and water and with a $12 \mathrm{~h}$ light/dark cycle. Genotyping was performed by PCR using DNA isolated from tail snips as described (Li et al. 2005; Sanchez et al. 2019). Animals did not carry the retinal degenerative mutations RD1 and RD8.

\section{STX3 variant detection}

Novel pathogenic STX3 mutations were identified in the five new individuals reported here (Table 1). STX3 mutations were identified by exome sequencing (WES) with DNA extracted from peripheral blood leukocytes in P3, P4 and P5 as described (Klee et al. 2020). For siblings P8 and P9, genome-wide autozygosity mapping analysis was performed with SNP array genotyping (HumanCytoSNP-12v2, Illumina), which highlighted the recently identified MVID gene, STX3. Subsequently, the coding exons and flanking intronic sequences corresponding to the STX3A transcript were directly sequenced (primer sequences are available from the authors on request). Variant designations are based on NCBI transcript reference NM_004177.5, using + 1 as the A of the ATG translation initiation codon. The DNA samples from all patients' parents, and from a number of additional family members were tested for the segregation and zygosity of STX3 variants identified in index patients (Supplementary Fig. 1).

We also present clinical follow-up data from five published individuals with STX3 loss-of-function variants; STX3 variant identification by WES was reported for P1 and P2 (Wiegerinck et al. 2014) for P6 (Maddirevula et al. 2019), P7 (Julia et al. 2019) and for P10 (Alsaleem et al.
2017). An STX3 transcript analysis was performed with leukocyte-derived RNA from P4, to assess the effect of a splice-site mutation in that family.

\section{Tissue preparation for immunohistology}

Following euthanasia, mouse retinae were collected, fixed and processed for immunohistochemistry as described (Liu et al. 2014). In brief, eyes were enucleated, the lens and cornea removed, and the resultant retinal eyecups immersion fixed in $4 \%$ para-formaldehyde in $0.1 \mathrm{M}$ sodium phosphate buffer $(\mathrm{pH}$ 7.4) at $4{ }^{\circ} \mathrm{C}$ for $24 \mathrm{~h}$. After fixation, eyes were cryoprotected in $30 \%$ sucrose/phosphate-buffered saline (PBS, pH 7.4) at $4{ }^{\circ} \mathrm{C}$ overnight and then embedded in OCT embedding medium (Tissue-Tek, Torrance, CA), fast-frozen in liquid nitrogen, and sectioned along the vertical meridian on a cryostat at a thickness of $18 \mu \mathrm{m}$. These sections were collected on Superfrost Plus microscope slides (Fisherbrand, Pittsburgh, PA) and stored at $-20{ }^{\circ} \mathrm{C}$ until use. Human retinal tissue was obtained from three female cadavers (ages 74, 75 and 85). Tissue was collected 4-6h postmortem. Human eyes were dissected, and a piece of the eyecup was fixed and sectioned as described above for the mouse tissue. For immunolabeling, sections were thawed immediately prior to use, rinsed, and incubated in blocking solution (5\% normal goat serum, and $0.3 \%$ Triton $\mathrm{X}-100$ in PBS) for $1 \mathrm{~h}$. All antibodies were diluted with blocking solution (5\% normal goat serum, and $0.3 \%$ Triton X-100 in PBS). Primary antibodies were applied at $4{ }^{\circ} \mathrm{C}$ overnight. After rinsing, secondary antibodies were applied for $1 \mathrm{~h}$. Secondary antibodies used at a dilution of 1:300. Double labeling using two primary antibodies raised in different host species was performed by simultaneously applying the primary antibodies and subsequently applying secondary antibodies conjugated to different fluorochromes simultaneously to visualize labeling. Sections were rinsed extensively and cover-slipped in a ProLong Gold antifade mounting medium with DAPI (Invitrogen, Eugene, OR).

\section{Antibodies}

The retinal distribution of STX3 was analyzed with polyclonal antibody (UT478, used at 1:300 dilution) (Liu et al. 2014) and with monoclonal antibody 12E5 (MilliporeSigma, Burlington, MA, United States), which recognize both STX3A and STX3B (Campbell et al. 2020). Monoclonal and polyclonal antibodies against specific markers were used as follows: Mouse monoclonal anti-rhodopsin (used at 1:500-1:1000), Millipore, Co., Temecula, CA (Molday and MacKenzie 1983); rabbit polyclonal anti red/green opsin (used at 1:200-1:500) Millipore Co., Temecula, CA(Otani et al. 2004); mouse monoclonal anti-CtBP2/Ribeye (used at1:200-1:400), Clone 16/CTBP2 BD Biosciences, San Jose, 


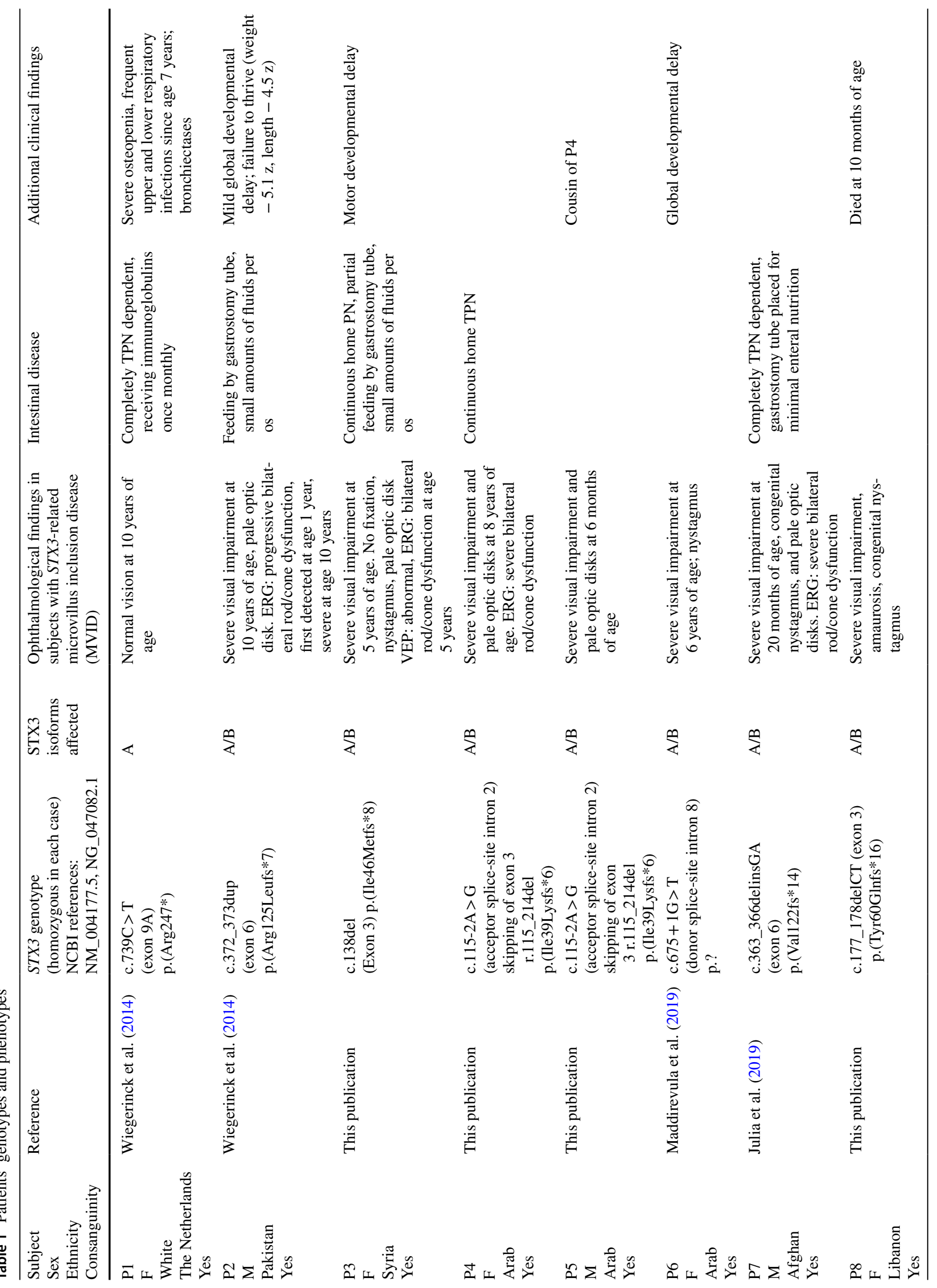




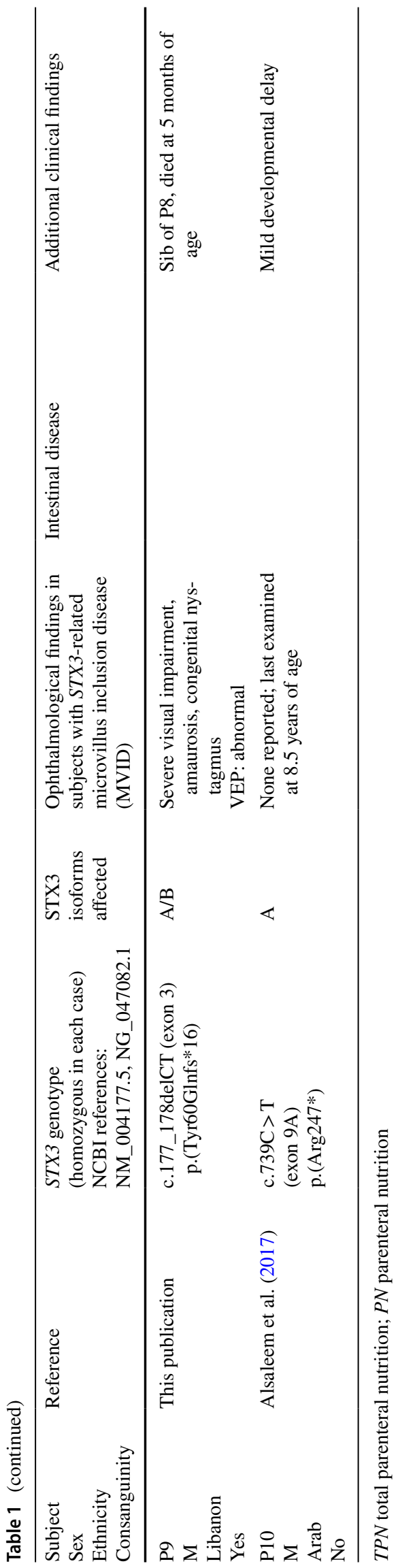

CA (against Mouse CtBP2 aa. 361-445), Syntaxin 3 mouse monoclonal clone 12E5, MilliporeSigma (Burlington, MA, United States) (used at 1:200 dilution).

Secondary goat-anti Ig and goat-anti rabbit mouse Ig conjugated to Cy3 (Jackson ImmunoResearch, West Grove, PA) or Alexa Fluor 488 (Molecular Probes, Eugene, OR) where used for labeling. All antibodies were diluted with blocking solution (5\% normal goat serum, and $0.3 \%$ Triton X-100 in PBS). Specificity of immunolabeling was confirmed by processing a second set of sections in the absence of the primary antibodies, or by substituting normal rabbit serum for polyclonal antibodies, as appropriate.

\section{Imaging}

Images were captured with a ZEISS LSM 800 confocal microscope (San Diego, CA) or with a Nikon A1R Confocal Laser Microscope (Hercules, CA) at a thickness of $0.3-0.5 \mu \mathrm{m}$. Confocal images were processed using the manufacturer's software Zen 2.5 (Blue edition) or Nikon Imaging Software Element (Version 4.20) and with Adobe Photoshop.

\section{Transcript expression analysis}

The expression of the different $S T X 3$ transcripts was analyzed by identifying matching reads from recent Illumina TruSeq ${ }^{\circledR}$ RNA-seq projects obtained with human retina samples (Ratnapriya et al. 2019). Datasets from retinas from six control donors selected for age (47-57 years) were analyzed using the BLAST program of the Sequence Read Archive database (SRA) at the NCBI (https://blast. ncbi.nlm.nih.gov/Blast.cgi?PROGRAM=blastn\&BLAST_ PROGRAMS $=$ megaBlast $\&$ PAGE_TYPE $=$ BlastSearch \& BLAST_SPEC $=$ SRA\&SHOW_DEFAULTS $=$ on) (individual specimen numbers: SRS3492913, SRS3493239, SRS3493240, SRS3493261, SRS3493276, SRS3493282). Probes of $120 \mathrm{bp}$ length covering $60 \mathrm{bp}$ upstream and downstream of the respective splice sites were analyzed. Default BLAST settings were used with the exception of the Expect threshold, which was set to 1000 . Reads were identified as matching if they had at least 90 bp identity with the probe, corresponding to an $E$ value of $<10^{-30}$. Reads per kilobase of transcript, per Million mapped reads (RPKM) where calculated for each probe.

Sequences of probes used:

HS STX3 8_9B

AGCAGCATCAAGGAGCTTCACGACATGTTTATGGAC ATCGCCATGCTGGTGGAGAATCAGGGATCCATGATT GACCGTATTGAGAACAACATGGACCAGTCAGTG GGCTTTGTGGAGCGG 
HS STX3 9B_10B:

GTGGAGCGGGCCGTGGCAGATACCAAAAAGGCT

GTCAAGTATCAGAGTGAAGCCCGGAGGAAGAAG ATCATGATCATGATCTGCTGTATTATCCTTGCGATC ATCTTAGCTTCCACCATT

HS STX3 8_9A:

AGCAGCATCAAGGAGCTTCACGACATGTTTATGGAC ATCGCCATGCTGGTGGAGAATCAGGGTGAGATGTTA GATAACATAGAGTTGAATGTCATGCACACAGTGGAC CACGTGGAGAAG

HS STX3 9A-10A:

AATAATCAATGCTAAAATGCCCAGCAACACAACTAC TAGCACAATGATAATTATCAATTTCTTCCGGGCCTG ACTCTGGTATTTCACAGCTTTTTTCGTTTCATCTCG TGCCTTCTCCAC

HS STX3 9A-11A:

GTGGAGAAGGCACGAGATGAAACGAAAAAAGCT GTGAAATACCAGAGTCAGGCCCGGAAGAAACTG ATTTCACTCCAGACTGGTGTGGCCACCCTTGTCTTC AGATGAGAATGGAGTCTG

\section{STX3 analysis of human tissues}

Human Tissue cDNA samples were purchased from Takara Bio USA, Inc. (Mountain View, CA). Standard PCR reactions were performed with the OneTaq Hot Start Quick kit (New England Biolabs) using these primers:

A: HS Syntaxin 3AB Sense exon 1:GGCTTCAGGATG AAGGACCG

B: HS Syntaxin 3B Sense Exon 7:GCAACCCGGCCA TCTTCACTTCTG

C: HS Syntaxin 3A Antisense exon 10A:AGCCCAACG GAAAGTCCAAT

D: HS Syntaxin 3B Antisense Exon 11B:CCTGTCCCT GTCCTCCGCCCAAT

GAPDH1: ATGACATCAAGAAGGTGGTG, GAPDH2: CATACCAGGAAATGAGCTTG

Conditions: 35 cycles for each reaction, annealing temperatures STX3: $55{ }^{\circ} \mathrm{C}$; GAPDH: $50{ }^{\circ} \mathrm{C}$. PCR reactions were analyzed using agarose gel-electrophoresis and correctness of the amplified fragments was confirmed by direct sequencing.

\section{Results}

\section{Pathogenic variants in the human STX3 gene that affect STX3B are associated with retinal disease}

Given the high level of STX3 expression in the murine and non-mammalian retina and roles for Stx 3 in protein trafficking in photoreceptors (Chuang et al. 2007; Kakakhel et al. 2020; Mazelova et al. 2009; Zulliger et al. 2015), and in neurotransmitter release at the first two synapses in the visual throughput pathway (Curtis et al. 2008, 2010; Datta et al. 2017; Liu et al. 2014) we asked whether MVID subjects with STX3 variants might have visual impairment. To address this question, we studied five newly identified MVID subjects in which we found homozygous STX3 variants; in addition, we investigated five previously described MVID subjects with STX3 variants (Alsaleem et al. 2017; Julia et al. 2019; Maddirevula et al. 2019; Wiegerinck et al. 2014) for the presence or absence of symptoms of marked visual impairment. In all affected individuals, the onset of diarrhea was in the first week of life, there was persisting diarrhea and histopathology demonstrated the characteristic features of MVID (not shown). The genotypes of each subject, along with visual testing results, are summarized in Table 1; Fig. 1a depicts the positions of all seven identified STX 3 variants, with each of the 10 subjects indicated. Supplementary Fig. 1 shows the pedigrees, segregation and chromatograms relating to novel STX3 variants. Importantly, each of these STX3 variants results in a premature stop codon, expected to either lead to nonsensemediated mRNA decay or to C-terminally truncated STX3 protein that lacks the transmembrane domain; the latter is thought to be essential for STX3 function (Rizo and Sudhof 2012). Thus, each of the identified human mutations is expected to behave as a loss-of-function mutation. Eight of the ten subjects (P2-P9) had pathogenic variants that were located in exons shared between the STX3A and the $S T X 3 B$ transcripts, and these eight subjects would be predicted to have loss of $S T X 3 A$ and $S T X 3 B$ expression. In contrast, two of the subjects (P1, P10) carried the same pathogenic variant (c.739C > T, p.Arg247*) that is located in exon 9A and thus spares STX3B.

Severe visual impairment was seen and a diagnosis of EOSRD rendered in individuals P2-P9, as evidenced by the inability to respond to or track visual stimuli, locate or reach for objects. Half the subjects also exhibited nystagmus. Five subjects underwent fundoscopic examination, revealing pallor of the optic disks (Fig. 1b, e, i, k). Unfortunately, because these subjects are both young and ill due to their MVID, with two sibling subjects (P8 and P9) succumbing to MVID-associated problems at less than one year of age, our ability to assess visual function 
more rigorously or conduct longitudinal studies was limited. In five subjects, electroretinography (ERG) demonstrated severe rod and cone dysfunction in both eyes. In the case of subject P2, ERG recordings with skin electrodes (Bradshaw et al. 2004) and fundus images were obtained at 1 year and at 10 years of age. The first ERG showed $\mathrm{A}$ and $\mathrm{B}$ waves that were greatly reduced in amplitude (Fig. 1c), consistent with a dramatic decrease in the light responses of photoreceptors and downstream bipolar cells, respectively. By 10 years of age, the A and B waves were almost entirely absent in this subject (Fig. 1d), suggesting a progressive loss in the light-sensing and signaling ability of the retina. Marked reductions in the A and B waves were also seen in P3 at 5 years of age (Fig. 1f-h). Subject $\mathrm{P} 7$, reported at age 15 months with nystagmus and visual impairment (Julia et al. 2019) had flat ERG recordings at 20 months of age indicating severe photoreceptor dysfunction (not shown). Subjects P8 and P9 had abnormal visual evoked potentials (VEP) responses. VEPs evaluate the integrity of the visual pathway from the retina to the visual cortex, but given the paucity of STX3 expression in the brain (see Fig. 11, m) these VEP abnormalities are most likely of retinal origin. By contrast, subjects P1 and P10 that carried the STX3A-only variant did not have visual impairment upon clinical inspection at 10 and at 8.5 years of age. Together, the ophthalmological findings obtained in MVID subjects point towards an essential role for STX3 in the retina.

\section{STX3B is a transcript selectively expressed in the human retina}

Given the visual deficits in MVID subjects with pathogenic variants in both $S T X 3 A$ and $S T X 3 B$, we asked whether both splice forms were present in the human retina. A GeneBank search identified a large number of ESTs and several fulllength cDNA clones corresponding to $S T X 3 A$. However, while no clones corresponding to $S T X 3 B$ were identified, analysis of the human STX3 gene sequence showed the presence of putative $S T X 3 B$-specific exons $9 \mathrm{~B}, 10 \mathrm{~B}$ and $11 \mathrm{~B}$ (Fig. 1a).

We find that the human STX3B transcript is generated by differential splicing in the same pattern as observed in the mouse, and expressed only in the retina (Fig. 1a, 1). STX3A and $S T X 3 B$ share exons 1-8 and differ with respect to exons 9-11. STX3A and STX3B differ in half of their SNARE and in their $\mathrm{C}$-terminal transmembrane domains.

For $S T X 3 A$, we detected strong signals in human small intestine, kidney, pancreas, placenta as well as in retina, with weaker expression levels detected in lung, liver and heart, and no signals above background detected in human brain and skeletal muscle tissues (Fig. 11). However, the commercial human retina cDNA samples (Takara Bio USA, Inc.) used in our experiments were obtained from pooled retinas from 99 donors and contained not only the neural retina, but also the retinal pigmented epithelial cell layer (RPE) (per manufacturer).

We next compared the relative levels of $S T X 3 A$ and $S T X 3 B$ mRNA in the human retina by analyzing the presence of splice junctions specific for the two isoforms in RNA-seq data sets from six normal human retina samples (Ratnapriya et al. 2019), and included a soluble form of STX3, STX3S that is generated by splicing together exons 9A and 11A and skipping exon 10A (Giovannone et al. 2018). These data demonstrate that $S T X 3 B$ is the major human retinal transcript (Fig. $1 \mathrm{~m}$ ).

\section{STX3 protein is located at retinal ribbon synapses and in the outer and inner segments of photoreceptors of the human retina}

To better understand the role of STX3 loss-of-function in our human cohort, we examined the expression and subcellular localization of the STX3 protein in the human retina via immunohistochemistry with STX3 antibodies that detect both STX3A and STX3B (Campbell et al. 2020; Liu et al. 2014; Zulliger et al. 2015). Given that our mRNA expression analysis of human retina showed that STX3A corresponds to only about $1 \%$ of total STX3 mRNA (Fig. 1m), the observed STX3 signal in human retina most likely corresponds almost exclusively to STX3B, as it does in the mouse retina (Curtis et al. 2008).

Photoreceptor terminals and bipolar cell terminals, located in the outer plexiform layer (OPL) and inner plexiform layer (IPL) respectively, labeled for the major structural protein of the synaptic ribbon, ribeye, exhibited strong STX3 immunolabeling (Fig. 2a-d), consistent with a role for STX3 in neurotransmitter release and the conveyance of visual information to downstream neurons in the human visual pathway. Ribeye shares a domain with the nuclear protein $\mathrm{CtBP} 2$, and thus $\mathrm{CtBP} 2$ antibodies label synaptic ribbons in addition to some nuclei, such as those in the inner nuclear layer (INL). Strong STX3 immunolabeling of photoreceptor inner and rhodopsin-labeled outer segments was observed in the human retina (Fig. $2 \mathrm{e}-\mathrm{h}$ ), contrasting with the labeling of only inner segments in mouse photoreceptors (Fig. 3) (Chuang et al. 2007; Zulliger et al. 2015). In addition, the inner and outer segments of cone photoreceptors also exhibited immunolabeling for STX3 in the human retina (Fig. 2i-1). The labeling of the plexiform layers and both inner and outer segments was observed with both STX3 antibodies and in retinal sections obtained from three different donors. 

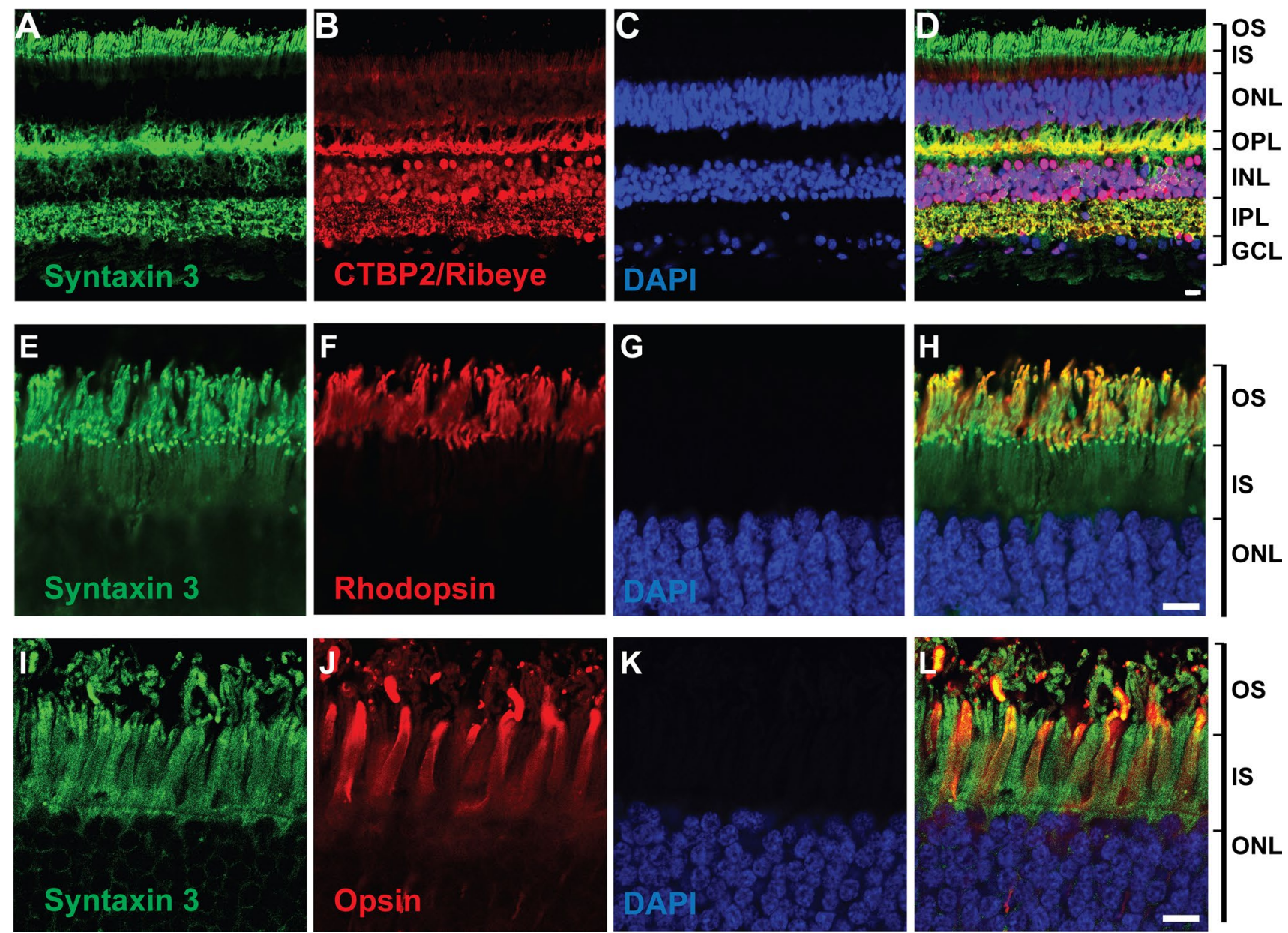

Fig. 2 STX3 is found in the synaptic terminals and the inner and outer segments of the photoreceptors and in bipolar cells in the human retina. Human retina was labeled with antibodies against STX3, CTBP2/Ribeye (a-d), rhodopsin (e-h), red/green cone opsin (i-l) and nuclei were counterstained with DAPI (nuclear marker).

Retinal layers are labeled on the right (Inner segments IS, outer segments OS, outer nuclear layer ONL, outer plexiform layer OPL, inner nuclear layer INL, inner plexiform layer IPL and ganglion cell layer GC). This is a representative example and similar results were obtained in $n=3$ donor samples. Scale bars: $20 \mu \mathrm{m}$

\section{Inactivation of the Stx3 gene in mouse rod photoreceptors leads to degeneration}

To probe for an essential role of Stx 3 in vision, we generated a cell-specific Stx 3 mouse knockout line, as complete inactivation of the Stx 3 gene in mice produces an embryonic lethal phenotype (Sanchez et al. 2019). A rod photoreceptor-specific inactivation of Stx 3 was achieved with a mouse line that expresses an optimized iCre under the control of the rhodopsin promoter (Li et al. 2005; Sanchez et al. 2019). Cre recombinase activity is first detectable in the retina of the Rho-iCre mice at 7 days of age, and it reaches a plateau at 18 days ( $\mathrm{Li}$ et al. 2005). Our breeding scheme generated the conditional Stx 3 knockout mice (fl/ $\mathrm{fl}$; Rho-cre/+), as well as homozygous Stx 3 floxed control mice $(\mathrm{fl} / \mathrm{fl} ;+/+)$ and heterozygous Cre expressing control mice $(+/+$; Rho-cre/ +$)$.

Whereas the retinal architecture, cell morphology, and number of cells in the nuclear layers appeared grossly normal at day 18, there was a reduction in Stx 3 immunolabeling in the OPL and in the inner segments of the photoreceptor layer of the fl/fl; Rho-cre/ + mice relative to controls (i.e. fl/ $\mathrm{fl}$ and Rho-Cre). Double-labeling for Stx3 and ribeye/CtBP2 and for Stx 3 and cone opsin revealed that the remaining Stx3 signal was in cone photoreceptors (Fig. 3b). Stx3 immunoreactivity appeared normal in the IPL, where the synaptic terminals of the bipolar cells reside (Fig. 3a).

At age 5 weeks, a $\approx 60 \%$ decrease in the thickness of the outer nuclear layer (ONL) and in the number of neuronal somata in the ONL was observed (Fig. 4a, c) indicating that a large number of photoreceptors had died. This demonstrated that $S t x 3$ expression is essential for the survival of retinal photoreceptors. At 5 weeks of age, Stx3 was still expressed in the cones in the rod-specific 

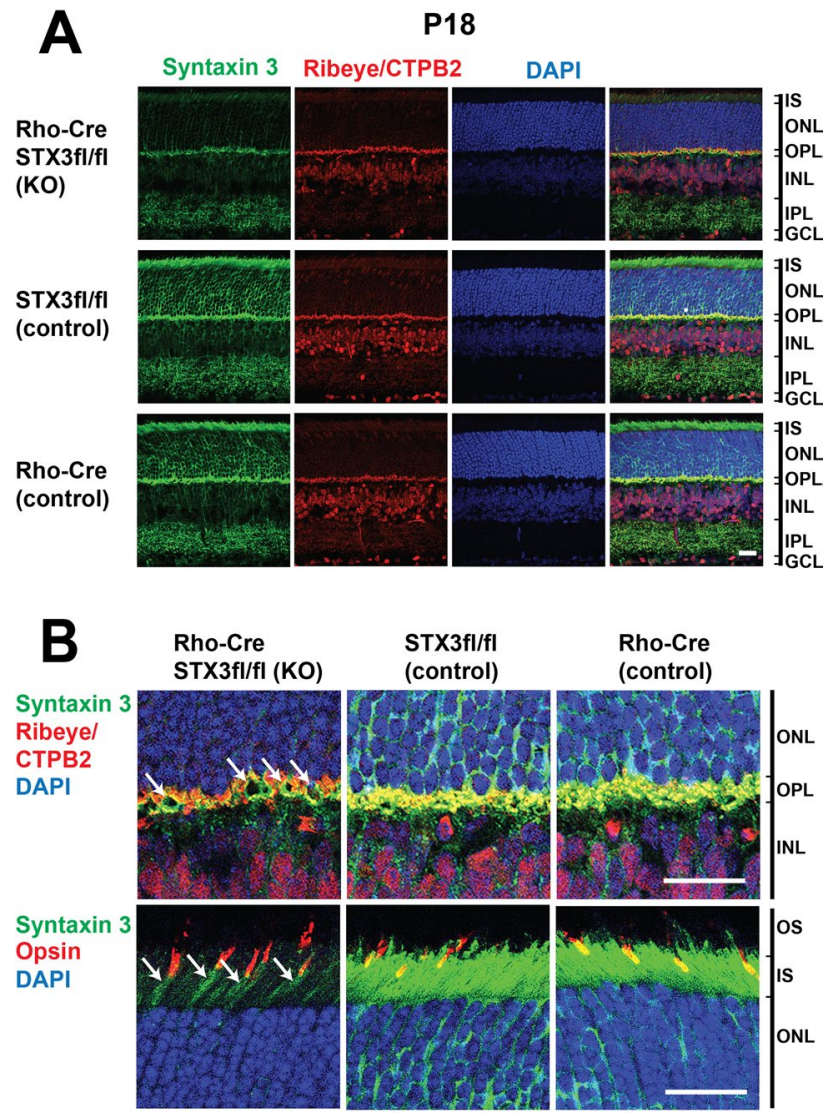

Fig. 3 At 18 days of age, conditional Stx3 knockout mice confirm an absence of Stx3 in rods with otherwise normal retinal morphology. a Representative vertical sections of retina from conditional Stx3 knockout mice (Rho-Cre/STX3fl/fl) and their controls were labeled with antibodies against Stx3, CtBP2/Ribeye and DAPI. Note normal retinal architecture but a lack of Stx3 immunolabeling of knockout mice in the IS, ONL and OPL. b Top panel, higher magnification of the OPL showing lack of Stx 3 protein label in the small rod terminals of the conditional knockout mice with Stx3 still present in the larger cone terminals marked by the arrows. Bottom panel. Labeling with antibodies against Stx3, red green opsin (cone marker) and DAPI confirms that the remaining Stx 3 expressing cells are cone photoreceptors. The arrows point to the remaining Stx3 signal found in the inner segments of cones. IS Inner segments, $O S$ outer segments, $O N L$ outer nuclear layer, $O P L$ outer plexiform layer, $I N L$ inner nuclear layer, $I P L$ inner plexiform layer, $G C L$ ganglion cell layer. Scale bars: $20 \mu \mathrm{m}$

conditional knockout animals, and the cone outer segments were morphologically grossly normal, with a small reduction in length and displacement of the outer segments and some ectopic localization of opsin to the OPL (Fig. 4a). In some of the remaining rods, rhodopsin was appropriately localized to the outer segments, but a marked amount of rhodopsin mislocalized to the OPL (Fig. 4a, arrows). These findings suggest that while rhodopsin can be trafficked to the ROS in the absence of Stx3, its proper trafficking in the rod is partially affected by the lack of Stx3.
At 8 and 12 weeks of age, an increasing cell loss and an increase in the ectopic expression of rhodopsin were observed. Cone photoreceptor loss and the ectopic expression of opsin in cones was also observed (Fig. 4b, c). The progressive degenerative phenotype was quantified by analyzing the cell numbers in the ONL and INL at different time points (Fig. 4c). This analysis revealed a rapid loss in the number of neuronal somata in the ONL, indicative of photoreceptor death, whereas the number of cells in the INL, wherein the somata of horizontal cells, bipolar cells and amacrine cells reside, was no different from controls.

\section{Discussion}

We report here that individuals with MVID and with biallelic STX3 loss-of-function variants that affect both STX3A and $S T X 3 B$ transcript isoforms display visual impairment consistent with an EOSRD. We show that $S T X 3 B$ rather than $S T X 3 A$ is highly expressed in the human retina, where the protein is found in the inner and outer segments of rod and cone photoreceptors, and in both plexiform layers, where the synaptic endings of photoreceptors and bipolar cells reside. Inactivation of Stx 3 in murine rod photoreceptors resulted in their rapid degeneration as shown here and in a very recent study (Kakakhel et al. 2020). The non-cell autonomous loss of cones in our knockout animals is likely attributable to a bystander effect, as seen in a number of retinal disorders (Brockerhoff and Fadool 2011; Lewis et al. 2010). Collectively, our findings in human subjects and mice establish that STX3 has an essential role in photoreceptor survival and vision.

In addition to visual impairment and MVID, global developmental delay was present in a few subjects with pathogenic STX3 variants (Chograni et al. 2015; Julia et al. 2019). The delay in patients from our case series is mild and might be largely attributable to the extensive hospitalizations, immobility caused by dependency on parenteral nutrition and severe visual impairment. Our examination of human brain samples did not indicate significant levels of STX3 expression overall, which is consistent with previous studies that have found the lowest level of STX3 expression in brain compared to other tissues in humans and rodents (Bennett et al. 1993; Curtis et al. 2008; Delgrossi et al. 1997). However, we cannot exclude the possibility that STX3 may be expressed in a small, specific subset of human brain cells, where it may impact human development or cognition. Early studies in mice suggested that STX3 may be involved in some types of learning and memory (Jurado et al. 2013), but a subsequent study of the same brain region failed to reveal an effect of STX3 inactivation on basal synaptic function or on a specific type of synaptic plasticity or learning and memory tasks (Shi et al. 2020). 
Fig. 4 Inactivation of Stx 3 in rod photoreceptors leads to progressive degeneration. a At 5 weeks of age there is a $\approx 60 \%$ reduction of photoreceptors in the knockout animals compared to littermate controls. Rhodopsin and cone opsin are still found in the outer segments, but aberrant distribution of rhodopsin is observed in the OPL and ONL of the knockout animals (arrows). b Analysis of older animals shows dramatic degeneration of photoreceptors after 8 and 12 weeks of age in the $\mathrm{KO}$ animals compared to controls. c Quantification of retinal layer cell numbers in the $\mathrm{KO}$ animals vs. controls over time shows the rapid specific degeneration of photoreceptors within several weeks of inactivation of Stx 3 in the rod photoreceptors at day 18. Each data point represents quantification of three randomly selected areas of the retina obtained from one animal with \pm SEM. Datapoints were fitted with the exponential plateau least squares fit method using PRISM 8 (Graphpad). The dramatic loss of photoreceptors was observed in six different knockout animals $>4$ weeks of age. IS Inner segments, $O S$ outer segments, $O N L$ outer nuclear layer, $O P L$ outer plexiform layer, $I N L$ inner nuclear layer, IPL inner plexiform layer, $G C L$ ganglion cell layer. Scale bars: $20 \mu \mathrm{m}$

A

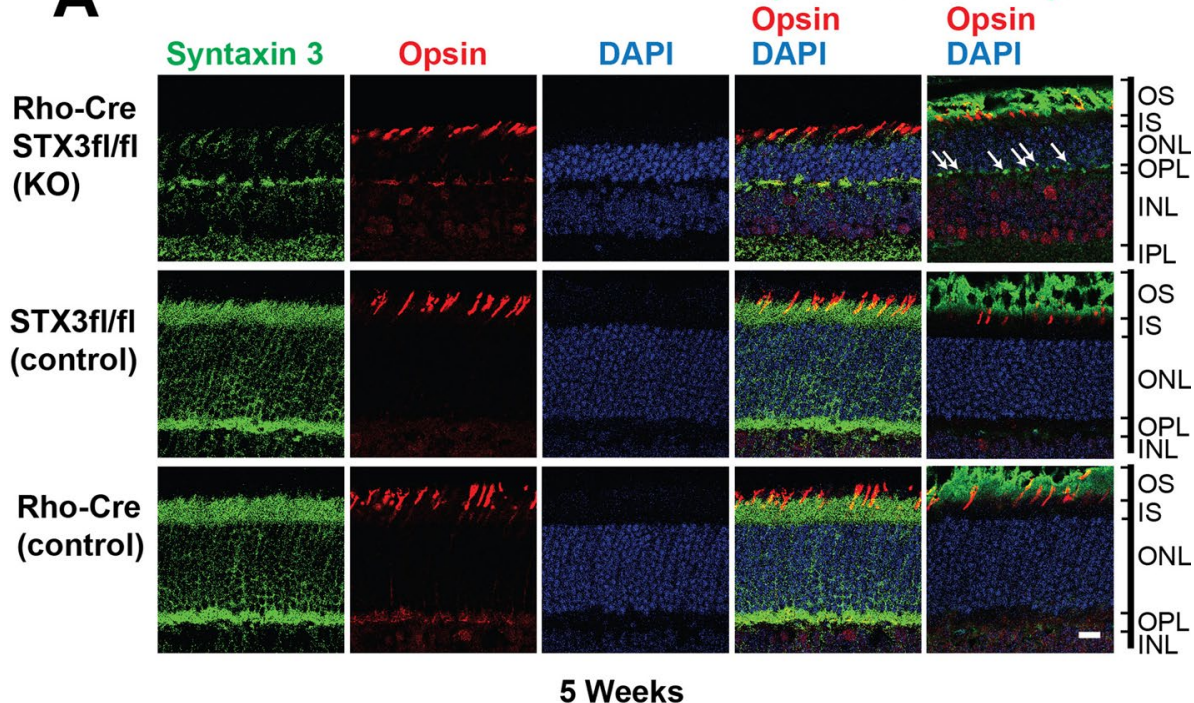

B

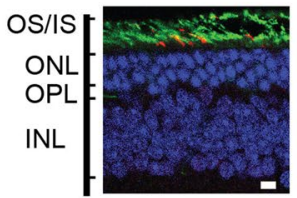

8 weeks Rho-Cre STX3fI/fl (KO)

C

12 weeks
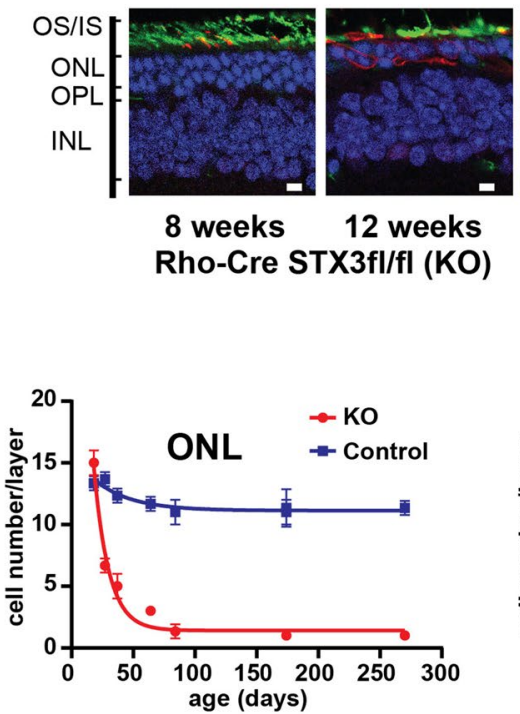

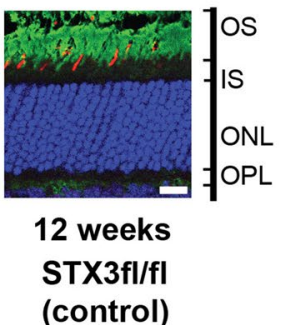

Rhodopsin Opsin DAPI

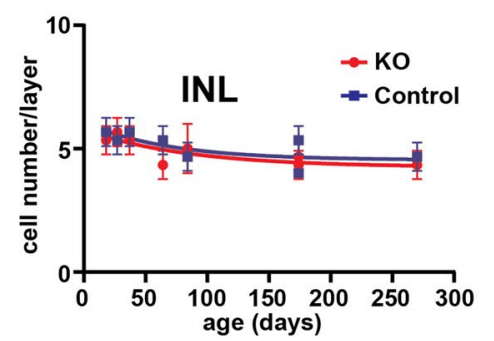

How might loss of STX3 function lead to photoreceptor degeneration? STX3 has been implicated in the trafficking of rhodopsin to the outer segments of photoreceptors (Chuang et al. 2007), and the proper trafficking of rhodopsin to its target compartment is essential for the survival of the photoreceptors. For example, pathogenic variants in the rhodopsin gene or in proteins that catalyze the trafficking of rhodopsin lead to degeneration of photoreceptors in humans and in animal models (Mendes et al. 2005). In addition, STX3 has been implicated in the trafficking of the essential outer segment proteins PRPH2 and ROM1 (Zulliger et al. 2015). However, we did not observe a mislocalization of rhodopsin in rods of P18 knockout mice, an age at which the Stx3 gene is expected to be fully inactivated ( $\mathrm{Li}$ et al. 2005). At age 5 weeks, however, rhodopsin was present in the outer segments and partly mislocalized to the OPL, indicating that proper rhodopsin trafficking is affected in the absence of Stx3. Similarly, a recent study using conditional knockout mouse lines that had Stx3 inactivated in rods as well as cones during early development, showed that rhodopsin, PRPH2 and ROM1 can get transported to the outer segments in the absence of Stx3 (Kakakhel et al. 2020). However, this study also found a significant amount of mislocalized rhodopsin, PRPH2 and ROM1 in photoreceptors that lack Stx3. The presence of mislocalized rhodopsin, PRPH2 and ROM1 might alter the physical properties, the composition, and the function of the affected compartments in photoreceptors, as suggested in the case of accumulation of truncated rhodopsin in various ectopic membrane locations (Deretic 2006). This pathological process could contribute to photoreceptor cell death by effecting protein degradation and by destabilizing the outer segment and its assembly, and by 
decreasing the availability of functional proteins important for the phototransduction process in the regions where the aberrant rhodopsin is present (Mendes et al. 2005).

Previously, our study of duodenal biopsies from patient P2 showed a complete loss of STX3 labeling, identified an abnormal subapical vesiculo-tubular network as abnormally extended, aberrant apical recycling endosomes, and indicated that disrupted trafficking between cargo vesicles and the apical plasma membrane is the primary cause of a defect of epithelial polarity and subsequent facultative loss of brush border integrity (Vogel et al. 2017). This membrane recycling defect supposedly leads to the symptoms of MVID, and supports hypotheses of trafficking or membrane recycling defects in photoreceptor cells of persons with STX3 deficiency.

STX3 in photoreceptor and bipolar cell synaptic endings has been suggested to play a critical role in synaptic transmission (Curtis et al. 2010; Datta et al. 2017; Hays et al. 2020). Conceivably, degeneration and death of photoreceptors could result from the disruption of neurotransmitter release from photoreceptors. However, in mouse models that inactivate the retina-specific $\mathrm{Ca}_{v} 1.4$ calcium channel, a protein essential for neurotransmitter release from rod photoreceptors, degeneration of photoreceptors is not observed (Mansergh et al. 2005). Thus, while loss of STX3 function may prevent the transmission of visual information from photoreceptors and bipolar cells to downstream neurons, there is no evidence to suggest that the loss of synaptic transmission itself is lethal to these neurons.

Syntaxins are also important for the trafficking of membrane itself and for membrane repair. STX3, in particular, has been shown to play a critical role in cell membrane expansion and neurite outgrowth (Darios and Davletov 2006). Together with the localization pattern of STX3 in human photoreceptors, this raises the possibility that STX3 may have a more general role in photoreceptor survival and the assembly and renewal of the human photoreceptor outer segment. This hypothesis is supported by studies that deleted or inactivated the plasma membrane syntaxins $1 \mathrm{~A}$ and $1 \mathrm{~B}$ from hippocampal (non-ciliated) neurons causing them to die in a manner unrelated to a defect in synaptic transmission (Peng et al. 2013; Vardar et al. 2016) and that the introduction of exogenous STX3 was able to rescue these neurons (Vardar et al. 2016). STX3 is the only plasma membraneassociated syntaxin expressed in photoreceptors (Sherry et al. 2006), and other syntaxins are only expressed at low levels (Shekhar et al. 2016). STX3 might participate in cellular house-keeping functions, and the observed mislocalization of outer segment proteins and synaptic phenotypes might represent, at least in part, an epi-phenomenon of dying photoreceptors.

The visual phenotype in humans that carry homozygous pathogenic STX3 variants might thus be caused by photoreceptor degeneration resulting from defective trafficking, from synaptic defects, or from disruption of more general cellular functions. Each of these must be considered when designing strategies for vision restoration for these subjects.

More than 200 genes associated with inherited photoreceptor degeneration have been identified in humans (Lee and Garg 2015), and their pathogenic variants cause diseases that can show differences in age of onset, course of the disease and additional (syndromic) organ involvement. Pathogenic variants affecting both the STX3A and STX3B isoforms cause a recognizable type of syndromic retinal disease, characterized by the unique combination of a congenital and intractable diarrhea and EOSRD. Before this study, the existence of the human syntaxin 3B had not been demonstrated, and it was not part of the annotated transcripts encoded by the human STX3 gene. Therefore, mutations affecting the STX3B transcript-specific exons may have been missed in previous large scale genomic screens for mutations implicated in human photoreceptor degeneration disorders. The STX3B-specific exons 9B and 10B code for essential regions of the syntaxin protein. Biallelic mutations in these retinaspecific exons would cause a new subtype of non-syndromic photoreceptor degeneration.

\section{Data and code availability}

The sequence of the $S T X 3 B$ transcript expressed in the retina that is generated by differential splicing has been deposited to GenBank (Accession number: MW273340). All the other data supporting the findings of this study are available within the article and from the corresponding authors upon request.

Supplementary Information The online version contains supplementary material available at https://doi.org/10.1007/s00439-021-02284-1.

Author contributions Conceptualization: ARJ, RH, RJ; Formal Analysis: ARJ, RH, RJ; Funding acquisition: ARJ, RH, RJ; Investigation: ARJ, XL, RA, SP, AV, VS, ML, ES, RA, MPS, USS, NM, KR, JE, AG, SAZ, FA, BA, MA, SA, FSA, LAH, TM, RH, RJ; Resources: LAH, TM; Software: ARJ, FSA; Visualization: DJ; Writing—original draft: ARJ., RH, RJWriting - review and editing: ARJ, XL, RA, SP, AV, VS, ML, ES, RA, MPS, USS, NM, KR, JE, AG, SAZ, FA, BA, MA, SA, FSA, LAH, TM, RH, RJ.

Funding Open access funding provided by University of Innsbruck and Medical University of Innsbruck. This work was supported by R01EY012128 (RH) and Core Grant P30EY028102. Additional funding was provided by the Vale-Asche Foundation via the Frederic B. Asche endowment (RH), and Österreichischen Nationalbank (grant no. 17968, ARJ). 


\section{Declarations}

Conflict of interest The authors declare no competing interests. This article was prepared while Roger Janz was employed at University of Texas Health Science Center in Houston. The opinions expressed in this article are the author's own and do not reflect the view of the National Institutes of Health, the Department of Health and Human Services, or the United States government.

Human and animal rights All human studies adhered to the principles set out in the Declaration of Helsinki. Animal procedures conformed to National Institutes of Health guidelines and were approved by the Animal Welfare Committee of the University of Texas Health Science Center at Houston.

Informed consent Informed consent was obtained from all participants as required by the IRB at the Medical University of Innsbruck, Austria. Individual-level data of human individuals, including clinical data, was de-identified and the authors received and archived written patient consent, with the exception of deceased patients.

Open Access This article is licensed under a Creative Commons Attribution 4.0 International License, which permits use, sharing, adaptation, distribution and reproduction in any medium or format, as long as you give appropriate credit to the original author(s) and the source, provide a link to the Creative Commons licence, and indicate if changes were made. The images or other third party material in this article are included in the article's Creative Commons licence, unless indicated otherwise in a credit line to the material. If material is not included in the article's Creative Commons licence and your intended use is not permitted by statutory regulation or exceeds the permitted use, you will need to obtain permission directly from the copyright holder. To view a copy of this licence, visit http://creativecommons.org/licenses/by/4.0/.

\section{References}

Alsaleem BMR, Ahmed ABM, Fageeh MA (2017) Microvillus inclusion disease variant in an infant with intractable diarrhea. Case Rep Gastroenterol 11:647-651. https://doi.org/10.1159/00047 9624

Bennett MK, Garcia-Arraras JE, Elferink LA, Peterson K, Fleming AM, Hazuka CD, Scheller RH (1993) The syntaxin family of vesicular transport receptors. Cell 74:863-873. https://doi.org/10. 1016/0092-8674(93)90466-4

Bradshaw K, Hansen R, Fulton A (2004) Comparison of ERGs recorded with skin and corneal-contact electrodes in normal children and adults. Doc Ophthalmol 109:43-55. https://doi.org/10. 1007/s10633-004-1751-3

Brockerhoff SE, Fadool JM (2011) Genetics of photoreceptor degeneration and regeneration in zebrafish. Cell Mol Life Sci 68:651-659. https://doi.org/10.1007/s00018-010-0563-8

Campbell JR, Li H, Wang Y, Kozhemyakin M, Hunt AJ Jr, Liu X, Janz R, Heidelberger R (2020) Phosphorylation of the retinal ribbon synapse specific t-SNARE protein syntaxin3B is regulated by light via a $\mathrm{Ca}(2+)$-dependent pathway. Front Cell Neurosci 14:587072. https://doi.org/10.3389/fncel.2020.587072

Chograni M, Alkuraya FS, Ourteni I, Maazoul F, Lariani I, Chaabouni HB (2015) Autosomal recessive congenital cataract, intellectual disability phenotype linked to STX3 in a consanguineous Tunisian family. Clin Genet 88:283-287. https://doi.org/10.1111/cge.12489
Chuang JZ, Zhao Y, Sung CH (2007) SARA-regulated vesicular targeting underlies formation of the light-sensing organelle in mammalian rods. Cell 130:535-547. https://doi.org/10.1016/j.cell.2007. 06.030

Curtis LB, Doneske B, Liu X, Thaller C, McNew JA, Janz R (2008) Syntaxin $3 b$ is a t-SNARE specific for ribbon synapses of the retina. J Comp Neurol 510:550-559. https://doi.org/10.1002/cne. 21806

Curtis L, Datta P, Liu X, Bogdanova N, Heidelberger R, Janz R (2010) Syntaxin $3 \mathrm{~B}$ is essential for the exocytosis of synaptic vesicles in ribbon synapses of the retina. Neuroscience 166:832-841. https:// doi.org/10.1016/j.neuroscience.2009.12.075

Darios F, Davletov B (2006) Omega-3 and omega-6 fatty acids stimulate cell membrane expansion by acting on syntaxin 3 . Nature 440:813-817. https://doi.org/10.1038/nature04598

Datta P, Gilliam J, Thoreson WB, Janz R, Heidelberger R (2017) Two pools of vesicles associated with synaptic ribbons are molecularly prepared for release. Biophys J 113:2281-2298. https://doi.org/10. 1016/j.bpj.2017.08.012

Delgrossi MH, Breuza L, Mirre C, Chavrier P, Le Bivic A (1997) Human syntaxin 3 is localized apically in human intestinal cells. J Cell Sci 110(Pt 18):2207-2214

Deretic D (2006) A role for rhodopsin in a signal transduction cascade that regulates membrane trafficking and photoreceptor polarity. Vision Res 46:4427-4433. https://doi.org/10.1016/j. visres.2006.07.028

Giovannone AJ, Winterstein C, Bhattaram P, Reales E, Low SH, Baggs JE, Xu M, Lalli MA, Hogenesch JB, Weimbs T (2018) Soluble syntaxin 3 functions as a transcriptional regulator. J Biol Chem. https://doi.org/10.1074/jbc.RA117.000874

Hays CL, Grassmeyer JJ, Wen X, Janz R, Heidelberger R, Thoreson WB (2020) Simultaneous release of multiple vesicles from rods involves synaptic ribbons and syntaxin 3B. Biophys J 118:967979. https://doi.org/10.1016/j.bpj.2019.10.006

Julia J, Shui V, Mittal N, Heim-Hall J, Blanco CL (2019) Microvillus inclusion disease, a diagnosis to consider when abnormal stools and neurological impairments run together due to a rare syntaxin 3 gene mutation. J Neonatal Perinatal Med 12:313-319. https://doi.org/10.3233/NPM-1852

Jurado S, Goswami D, Zhang Y, Molina AJ, Sudhof TC, Malenka RC (2013) LTP requires a unique postsynaptic SNARE fusion machinery. Neuron 77:542-558. https://doi.org/10.1016/j.neuron.2012.11.029

Kakakhel M, Tebbe L, Makia MS, Conley SM, Sherry DM, A1Ubaidi MR, Naash MI (2020) Syntaxin 3 is essential for photoreceptor outer segment protein trafficking and survival. Proc Natl Acad Sci USA 117:20615-20624. https://doi.org/10.1073/ pnas. 2010751117

Klee KMC, Janecke AR, Civan HA, Rosipal S, Heinz-Erian P, Huber LA, Muller T, Vogel GF (2020) AP1S1 missense mutations cause a congenital enteropathy via an epithelial barrier defect. Hum Genet 139:1247-1259. https://doi.org/10.1007/ s00439-020-02168-w

Lee K, Garg S (2015) Navigating the current landscape of clinical genetic testing for inherited retinal dystrophies. Genet Med 17:245-252. https://doi.org/10.1038/gim.2015.15

Lewis A, Williams P, Lawrence O, Wong RO, Brockerhoff SE (2010) Wild-type cone photoreceptors persist despite neighboring mutant cone degeneration. J Neurosci 30:382-389. https://doi.org/10. 1523/JNEUROSCI.5019-09.2010

Li S, Chen D, Sauve Y, McCandless J, Chen YJ, Chen CK (2005) Rhodopsin-iCre transgenic mouse line for Cre-mediated rod-specific gene targeting. Genesis 41:73-80. https://doi.org/10.1002/ gene. 20097

Liu X, Heidelberger R, Janz R (2014) Phosphorylation of syntaxin $3 \mathrm{~B}$ by CaMKII regulates the formation of t-SNARE complexes. 
Mol Cell Neurosci 60:53-62. https://doi.org/10.1016/j.mcn.2014. 03.002

Low SH, Chapin SJ, Wimmer C, Whiteheart SW, Komuves LG, Mostov KE, Weimbs T (1998) The SNARE machinery is involved in apical plasma membrane trafficking in MDCK cells. J Cell Biol 141:1503-1513. https://doi.org/10.1083/jcb.141.7.1503

Maddirevula S, Alzahrani F, Al-Owain M, Al Muhaizea MA, Kayyali HR, AlHashem A, Rahbeeni Z, Al-Otaibi M, Alzaidan HI, Balobaid A, El Khashab HY, Bubshait DK, Faden M, Yamani SA, Dabbagh O, Al-Mureikhi M, Jasser AA, Alsaif HS, Alluhaydan I, Seidahmed MZ, Alabbasi BH, Almogarri I, Kurdi W, Akleh H, Qari A, Al Tala SM, Alhomaidi S, Kentab AY, Salih MA, Chedrawi A, Alameer S, Tabarki B, Shamseldin HE, Patel N, Ibrahim N, Abdulwahab F, Samira M, Goljan E, Abouelhoda M, Meyer BF, Hashem M, Shaheen R, AlShahwan S, Alfadhel M, Ben-Omran T, Al-Qattan MM, Monies D, Alkuraya FS (2019) Autozygome and high throughput confirmation of disease genes candidacy. Genet Med 21:736-742. https://doi.org/10.1038/ s41436-018-0138-x

Mansergh F, Orton NC, Vessey JP, Lalonde MR, Stell WK, Tremblay F, Barnes S, Rancourt DE, Bech-Hansen NT (2005) Mutation of the calcium channel gene Cacnalf disrupts calcium signaling, synaptic transmission and cellular organization in mouse retina. Hum Mol Genet 14:3035-3046. https://doi.org/10.1093/hmg/ ddi336

Mazelova J, Ransom N, Astuto-Gribble L, Wilson MC, Deretic D (2009) Syntaxin 3 and SNAP-25 pairing, regulated by omega-3 docosahexaenoic acid, controls the delivery of rhodopsin for the biogenesis of cilia-derived sensory organelles, the rod outer segments. J Cell Sci 122:2003-2013. https://doi.org/10.1242/jcs. 039982

Mendes HF, van der Spuy J, Chapple JP, Cheetham ME (2005) Mechanisms of cell death in rhodopsin retinitis pigmentosa: implications for therapy. Trends Mol Med 11:177-185. https://doi.org/ 10.1016/j.molmed.2005.02.007

Molday RS, MacKenzie D (1983) Monoclonal antibodies to rhodopsin: characterization, cross-reactivity, and application as structural probes. Biochemistry 22:653-660. https://doi.org/10.1021/bi002 $72 \mathrm{a} 020$

Otani A, Dorrell MI, Kinder K, Moreno SK, Nusinowitz S, Banin E, Heckenlively J, Friedlander M (2004) Rescue of retinal degeneration by intravitreally injected adult bone marrow-derived lineagenegative hematopoietic stem cells. J Clin Invest 114:765-774. https://doi.org/10.1172/JCI21686

Peng L, Liu H, Ruan H, Tepp WH, Stoothoff WH, Brown RH, Johnson EA, Yao WD, Zhang SC, Dong M (2013) Cytotoxicity of botulinum neurotoxins reveals a direct role of syntaxin 1 and SNAP-25 in neuron survival. Nat Commun 4:1472. https://doi.org/10.1038/ ncomms 2462

Ratnapriya R, Sosina OA, Starostik MR, Kwicklis M, Kapphahn RJ, Fritsche LG, Walton A, Arvanitis M, Gieser L, Pietraszkiewicz A, Montezuma SR, Chew EY, Battle A, Abecasis GR, Ferrington DA, Chatterjee N, Swaroop A (2019) Retinal transcriptome and eQTL analyses identify genes associated with age-related macular degeneration. Nat Genet 51:606-610. https://doi.org/10.1038/ s41588-019-0351-9

Rizo J, Sudhof TC (2012) The membrane fusion enigma: SNAREs, Sec1/Munc18 proteins, and their accomplices-guilty as charged? Annu Rev Cell Dev Biol 28:279-308. https://doi.org/10.1146/ annurev-cellbio-101011-155818

Robichaux MA, Potter VL, Zhang Z, He F, Liu J, Schmid MF, Wensel TG (2019) Defining the layers of a sensory cilium with STORM and cryoelectron nanoscopy. Proc Natl Acad Sci USA 116:2356223572. https://doi.org/10.1073/pnas. 1902003116

Sanchez E, Gonzalez EA, Moreno DS, Cardenas RA, Ramos MA, Davalos AJ, Manllo J, Rodarte AI, Petrova Y, Moreira DC, Chavez MA, Tortoriello A, Lara A, Gutierrez BA, Burns AR, Heidelberger R, Adachi R (2019) Syntaxin 3, but not syntaxin 4 , is required for mast cell-regulated exocytosis, where it plays a primary role mediating compound exocytosis. J Biol Chem 294:3012-3023. https://doi.org/10.1074/jbc.RA118.005532

Shekhar K, Lapan SW, Whitney IE, Tran NM, Macosko EZ, Kowalczyk M, Adiconis X, Levin JZ, Nemesh J, Goldman M, McCarroll SA, Cepko CL, Regev A, Sanes JR (2016) Comprehensive classification of retinal bipolar neurons by single-cell transcriptomics. Cell 166(1308-1323):e30. https://doi.org/10.1016/j.cell.2016.07. 054

Sherry DM, Mitchell R, Standifer KM, du Plessis B (2006) Distribution of plasma membrane-associated syntaxins 1 through 4 indicates distinct trafficking functions in the synaptic layers of the mouse retina. BMC Neurosci 7:54. https://doi.org/10.1186/ 1471-2202-7-54

Shi S, Ma K, Bin NR, Harada H, Xie X, Huang M, Liu H, Lee S, Wang XF, Adachi R, Monnier PP, Zhang L, Sugita S (2020) Syntaxin-3 is dispensable for basal neurotransmission and synaptic plasticity in postsynaptic hippocampal CA1 neurons. Sci Rep 10:709. https://doi.org/10.1038/s41598-019-57388-6

Vardar G, Chang S, Arancillo M, Wu YJ, Trimbuch T, Rosenmund C (2016) Distinct functions of syntaxin-1 in neuronal maintenance, synaptic vesicle docking, and fusion in mouse neurons. J Neurosci 36:7911-7924. https://doi.org/10.1523/JNEUROSCI. 1314-16.2016

Vogel GF, Klee KM, Janecke AR, Muller T, Hess MW, Huber LA (2015) Cargo-selective apical exocytosis in epithelial cells is conducted by Myo5B, Slp4a, Vamp7, and Syntaxin 3. J Cell Biol 211:587-604. https://doi.org/10.1083/jcb.201506112

Vogel GF, Hess MW, Pfaller K, Huber LA, Janecke AR, Muller T (2016) Towards understanding microvillus inclusion disease. Mol Cell Pediatr 3:3. https://doi.org/10.1186/s40348-016-0031-0

Vogel GF, Janecke AR, Krainer IM, Gutleben K, Witting B, Mitton SG, Mansour S, Ballauff A, Roland JT, Engevik AC, Cutz E, Muller T, Goldenring JR, Huber LA, Hess MW (2017) Abnormal Rab11Rab8-vesicles cluster in enterocytes of patients with microvillus inclusion disease. Traffic 18:453-464. https://doi.org/10.1111/ tra. 12486

Wiegerinck CL, Janecke AR, Schneeberger K, Vogel GF, van Haaften-Visser DY, Escher JC, Adam R, Thoni CE, Pfaller K, Jordan AJ, Weis CA, Nijman IJ, Monroe GR, van Hasselt PM, Cutz E, Klumperman J, Clevers H, Nieuwenhuis EE, Houwen RH, van Haaften G, Hess MW, Huber LA, Stapelbroek JM, Muller T, Middendorp S (2014) Loss of syntaxin 3 causes variant microvillus inclusion disease. Gastroenterology 147(65-68):e10. https://doi. org/10.1053/j.gastro.2014.04.002

Zulliger R, Conley SM, Mwoyosvi ML, Stuck MW, Azadi S, Naash MI (2015) SNAREs interact with retinal degeneration slow and rod outer segment membrane protein-1 during conventional and unconventional outer segment targeting. PLoS ONE 10:e0138508. https://doi.org/10.1371/journal.pone.0138508

Publisher's Note Springer Nature remains neutral with regard to jurisdictional claims in published maps and institutional affiliations. 


\section{Authors and Affiliations}

Andreas R. Janecke ${ }^{1,2}$ (D) $\cdot$ Xiaoqin Liu $^{3} \cdot$ Rüdiger Adam $^{4} \cdot$ Sumanth Punuru ${ }^{3} \cdot$ Arne Viestenz $^{5}$ (D) Valeria Strau $\aleph^{6}$. Martin Laass ${ }^{7}$. Elizabeth Sanchez ${ }^{8} \cdot$ Roberto Adachi $^{8}$ ID $\cdot$ Martha P. Schatz $^{9}$ - Ujwala S. Saboo ${ }^{9}$. Naveen Mittal ${ }^{10}$. Klaus Rohrschneider ${ }^{11}$ (D) Johanna Escher ${ }^{12}$ (D) . Anuradha Ganesh ${ }^{13}$. Sana Al Zuhaibi ${ }^{13} \cdot$ Fathiya Al Murshedi $^{14}$.

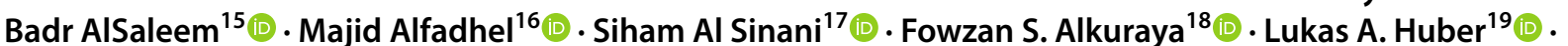
Thomas Müller $^{1} \cdot$ Ruth Heidelberger $^{3}$ (D) $\cdot$ Roger Janz $^{3,20}$ (D)

1 Department of Pediatrics I, Medical University of Innsbruck, Anichstrasse 35, 6020 Innsbruck, Austria

2 Division of Human Genetics, Medical University of Innsbruck, Innsbruck, Austria

3 Department of Neurobiology and Anatomy, MSB 7.046, McGovern Medical School at the University of Texas HSC (UTHealth), 6431 Fannin Street, Houston, TX 77030, USA

4 University Children's Hospital, Medical Faculty Mannheim, Heidelberg University, 68167 Mannheim, Germany

5 Department of Ophthalmology, University Medical Center Halle, Martin-Luther-University Halle-Wittenberg, Halle, Germany

6 Klinik für Kinder- und Jugendmedizin, Universitätsklinikum Halle, Halle, Germany

7 Klinik und Poliklinik f. Kinder- u. Jugendmedizin, University of Dresden, Dresden, Germany

8 Department of Pulmonary Medicine, Division of Internal Medicine, The University of Texas MD Anderson Cancer Center, Houston, TX, USA

9 Department of Ophthalmology, University of Texas Health Science Center, San Antonio, TX, USA

10 Department of Department of Pediatrics, Division of Pediatric Gastroenterology, University of Texas Health Science Center, San Antonio, TX, USA
11 Augenklinik, Universitätsklinikum Heidelberg, Heidelberg, Germany

12 Erasmus MC-Sophia Children's Hospital, Rotterdam, The Netherlands

13 Department of Ophthalmology, Sultan Qaboos University Hospital, Muscat, Oman

14 Genetic and Developmental Medicine Clinic, Sultan Qaboos University Hospital, Muscat, Oman

15 King Fahad Medical City, Children's Specialized Hospital, Riyadh, Saudi Arabia

16 Genetics Division and Medical Genomic Research Lab, King Saud Bin Abdulaziz University for Health Sciences (KSAU-HS), Riyadh, Saudi Arabia

17 Department of Child Health, Sultan Qaboos University Hospital, Muscat, Oman

18 Department of Genetics, King Faisal Specialist Hospital and Research Center, Riyadh, Saudi Arabia

19 Division of Cell Biology, Medical University of Innsbruck, Innsbruck, Austria

20 Present Address: Center for Scientific Review, National Institutes of Health, Bethesda, MD, USA 